\title{
Efficacy of Oral Propranolol on the Treatment of Retinopathy of Prematurity
}

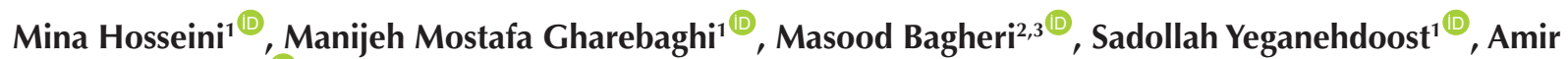 \\ Eftekhari Milani $^{* * \mathbb{}}$, Mohamad Reza Niyousha ${ }^{3}$
}

\begin{abstract}
Objectives: Due to recent advances in caring for premature neonates, premature retinopathy has become an important cause of preventable blindness and ocular disabilities. Therefore, more attention is paying to its diagnostic-therapeutic methods. Thus, the current trial assessed the efficacy of oral propranolol on the treatment of retinopathy of prematurity (ROP) in neonates.

Materials and Methods: In the current trial, 58 neonates with ROP were included and the efficacy of oral propranolol $(0.5 \mathrm{mg} / \mathrm{kg}$ every 8 hours) was compared with the control group.

Results: In the intervention group, the stage of ROP was almost the same as the control group while $31.1 \%$ of neonates in the control group were progressed to stage 3. On the other hand, it was only $6.9 \%(P=0.009)$ in the intervention group. In patients receiving propranolol, only 2 cases required invasive therapeutic interventions whereas 9 patients required such interventions in the control group $(P=0.009)$. The ROP improvement rate was significantly higher in the intervention group. In the control group, the overall progression to higher stages was about $48.3 \%$ while it was $13.8 \%$ for the intervention group. The mean age of neonates at the time of retinal artery completion was $91.40 \pm 22.16$ and $128.08 \pm 79.89$ days for the control and intervention groups, respectively $(P=0.136)$.

Conclusions: The results of this study showed that adding oral propranolol to standard therapy can reduce the rate of ROP progression to higher stages in preterm neonates with low birth weight, and the need for invasive treatments.

Keywords: Propranolol, Avastin, Laser therapy, Retinopathy of prematurity
\end{abstract}

\section{Introduction}

Premature birth is the cause of one-third of neonatal mortality, $45 \%$ of cerebral palsy, $35 \%$ of visual impairment, and $25 \%$ of behavioral or auditory impairment in American children (1). The retinopathy of prematurity (ROP), which was first described by Terry in 1942 as "Terry syndrome" (1), is the significant reason for blindness in childhood (2). The fetal retinal arteries begin to grow at 16 weeks of gestation and reach the nasal and temporal ora serrata at weeks 36 and 40, respectively. Hence, highly premature neonates have incomplete retinal arteries, which, in severe cases, can lead to visual impairment (1).

In recent years, due to advancements in neonatal care methods in intensive care units, ROP has become the prevalent reason for sight-threatening and ocular disabilities in surviving premature neonates so that its rates are estimated from $5 \%$ in developed countries to $30 \%$ in developing countries (3). Approximately 400 cases of blindness and 4300 cases of severe retinal scarring due to premature retinopathy are annually estimated in the United States (4). The significant risk factors for the incidence and progression of premature retinopathy are preterm delivery particularly $<32$ weeks gestational age
(GA) or birth weight (BW) less than $1500 \mathrm{~g}$ (5) so that more than $80 \%$ of ROP cases are in premature neonates with a weight less than $1000 \mathrm{~g}$ (3). Other risk factors include respiratory disorders, infection, heart diseases, bradycardia, transfusion, oxygen intake, duration of ventilation, and the like (3).

The vascular endothelial growth factor (VEGF) is the major factor in ROP pathophysiology. This is a pivotal mediator for the normal completion of retinal blood vessels. However, abnormally high VEGF levels in the retina and vitreous are primarily the main cause of developing ROP (6). ROP has two main development phases (7). In the first phase, VEGF levels decrease due to hyperoxic conditions following postnatal oxygenation occurring after the delivery, which leads to insufficient growth of retinal vessels. At the next phase, which is named as the proliferation phase and begins at about the 32nd week, VEGF levels start to increase due to the ischemic retina. VEGF may also increase other growth factors including insulin-like growth factor 1 (IGF-1) which, in turn, produces more VEGF through positive feedback (8). These conditions lead to the neovascularization of the retina and the creation of abnormal vessels that can cause 


\section{Key Messages}

- The administration of oral propranolol can reduce the rate of ROP progression and the need for invasive treatments, and shortens the completion period of retinal vascularization in premature neonates with low BW. However, the benefits of prescribing should be weighed against the possible side effects.

bleeding and subsequent problems, resulting in tractional retinal detachment (9).

ROP is usually asymptomatic, thus suspected neonates need screening. Based on the American Academy of Pediatrics guidelines, the recommended screening for ROP should be performed for neonates with a GA $<30$ weeks or BW $\leq 1500 \mathrm{~g}$, as well as neonates with a BW 1500-2000 g or GA more than 30 weeks with inappropriate conditions such as those who need cardiorespiratory support or are at high risk for retinopathy according to the neonatologist's diagnosis. Based on the GA, eye examinations should be performed between 31 and 33 weeks of GA or 4 and 6 weeks after the delivery (9). The severity of ROP is classified into three zones and five stages (Table 1).

Many stage- 2 neonates recover without any treatment while more severe cases need treatment. Treatment at stage 3 will have a significant effect on visual prognosis, and failure to treat leads to the progression of the disease and even blindness (11). Extensive studies have so far been conducted in the field of diagnosis and treatment of the ROP. Recently, with the introduction of VEGF as a major mediator in the pathophysiology of the ROP, the intravitreal injection of anti-VEGF factors as an appropriate treatment has substituted destructive methods such as laser therapy $(3,4)$.

Although new therapies have improved the prognosis of neonates with ROP, the rate of adverse outcomes is still high (12). Neonates need anesthesia in equipped and advanced centers. Considering the possibility of complications such as vitreous hemorrhage and corneal opacity in laser treatment, as well as the adverse outcomes of intravitreal anti-VEGF injection (including invasive treatment, cataract, endophthalmitis, and the like), less invasive, inexpensive, and affordable therapies have been emphasized in recent years. Recent studies have shown that the administration of oral propranolol may be effective in improving ROP by reducing VEGF and IGF-1 levels $(3,13)$.

Based on the hypothesis indicating that the administration of beta-blockers can reduce the expression of angioproliferative factors (including VEGF and IGF1) through beta-2 adrenoceptors blockage (13), the current study was designed to evaluate the preventive and therapeutic effects of oral propranolol in the management of ROP patients.

\section{Materials and Methods}

This double-blind, randomized controlled clinical trial (RCT) was performed for 12 months on preterm neonates
Table 1. The Five Stages of ROP Progression

\begin{tabular}{ll}
\hline Stage $\mathbf{1}$ & Demarcation line \\
\hline Stage $\mathbf{2}$ & Ridge \\
\hline Stage 3 & Ridge with extraretinal fibrovascular proliferation \\
\hline Stage 4A & Extrafoveal retinal detachment \\
\hline Stage 4B & Partial retinal detachment including the fovea \\
\hline Stage 5 & Total retinal detachment \\
\hline Note. ROP: Retinopathy of prematurity. \\
Source: Schachat et al (10).
\end{tabular}

admitted to Alzahra hospital in Tabriz, Iran. All premature neonates with a $\mathrm{GA}<30$ weeks and a $\mathrm{BW}<1.250$ gr underwent ophthalmological examination. Among them, patients with stage 1 or 2 ROP were included in the study after obtaining informed consent from their parents. On the other hand, patients with cardiovascular disease, ocular abnormalities, acute renal failure, cerebral hemorrhage, major congenital or chromosomal abnormalities, and hemodynamic instability were excluded from the study. During the study, patients were excluded in the case of side effects such as hypotension, bradycardia ( $<80$ beats/ minute), and hypoglycemia $(<40 \mathrm{mg} / \mathrm{dL})$.

Among the premature neonates who were eligible for the study and whose ocular examination revealed the ROP, 58 cases were allocated to one of the two intervention or control groups using a random number table. It worth noting that all ophthalmological examinations were performed by a trained ophthalmologist who was not involved in the medical treatment of neonates and was unaware of the administered drugs. Neonates were grouped based on the administration of propranolol, and the nurse who was recording the patients' information was unaware of the drug that was planned for administration and the grouping.

After identifying the patients, oral propranolol with a dose of $0.5 \mathrm{mg} / \mathrm{kg}$ every 8 hours was added to the standard treatment in the study group, and the second group only received the standard treatment. Oral propranolol treatment continued until the complete treatment of retinal perfusion, complete elimination of ROP, or maximally up to 90 days. In the propranolol group, blood sugar was measured before administration, one hour later, and then every 6 hours during the first 24 hours of treatment. For neonates who were receiving oxygen, pulse oximetry was performed during the treatment, and the oxygen level was adjusted in the range of $90 \%-95 \%$. Daily blood pressure monitoring was performed as well. Every two weeks, liver tests and complete blood count were monitored, along with other neonatal examinations, and ophthalmoscopy was performed periodically once a week. After discharging the neonates, the occurrence of any problem such as cyanosis attacks, paleness or lethargy, and the lack of proper nutrition was followed up through telephone calls.

The two groups were compared in terms of the rate 
of improvement or progression to higher levels of ROP, the number of follow-up days until the recovery, and the need for invasive treatments including laser therapy and the intravitreal injection of Avastin, advanced surgical interventions, and subsequent vision problems. Data were analyzed using IBM SPSS software (version 13, SPSS, Chicago, IL). Descriptive statistical methods, nonindependent t-test, and Chi-square test were used to analyze the data, and a $P<0.05$ was considered statistically significant.

Oral propranolol is a low-risk, inexpensive, and affordable drug with limited side effects. Before starting the research, written informed consent was taken from the parents of neonates and they were assured of voluntary participation and data confidentiality during the study implementation. In addition, no additional cost was imposed on the participants, and the participating neonates were not deprived of standard therapeutic interventions.

\section{Results}

In general, 58 neonates with ROP were included in this RCT. Neonates were divided into intervention and control groups. The two groups were not significantly different in terms of the GA, BW, APGAR score at birth and 5 minutes later, as well as maternal risk factors (e.g., diabetes, premature rupture of membranes, chorioamnionitis, hypertension, hypothyroidism, HELLP, and the like) and the treatments received before, during, and after the birth (Table 2). The mean age of ROP diagnosis in the patients of the intervention and control groups was $47.27 \pm 1091$ and $50.86 \pm 16.40$ days, respectively $(P=0.233)$. Moreover, the mean neonatal weight at the time of diagnosing ROP in the patients of the intervention and control groups was $1529.68 \pm 388.16$ and $1614.09 \pm 180.24$, respectively, representing no statistically significant difference between the two groups $(P=0.824)$. In the intervention group, $13(44.9 \%)$ and $16(55.1 \%)$ patients were in stages 1 and 2 of the disease, respectively, while these values were 14 (48.2\%) and 15 (51.8\%) for the control group, respectively $(P=0.217)$. The characteristics of patients with ROP are described in Table 3. As shown, the two groups were not significantly different in terms of the disease severity.

The youngest and oldest participants were 28 and 57 days. Further, the mean duration of propranolol administration in the intervention group was $20.89 \pm 12.44$ days. At the end of the study, the progression from stage 1 to 2 and stage 2 to 3 ROP was observed in $5(35.7 \%)$ and $9(60.0 \%)$ cases in the control group, as well as 2 $(15.38 \%)$ and $2(12.5 \%)$ patients in the intervention group ( $P=0.001,0.009)$, respectively. According to the results, the progression rate of ROP in the intervention group was significantly lower compared to the control group so that the overall progression to a higher stage was nearly $48.3 \%$ in the control group while it was $13.8 \%$ in the intervention group (Table 4). Finally, 17 patients needed ophthalmic surgical intervention to treat the ROP, of which 4 (13.8\%) cases were in the intervention group (two laser therapy and two intravitreal Avastin injection cases) and 13 (44.8\%) of them belonged to the control group including eight laser therapy and five intravitreal Avastin injection cases $(P=0.001)$.

In the subgroup analysis, the participants were divided into two groups including those receiving definitive ophthalmic surgical intervention for ROP (laser therapy or intravitreal Avastin injection, $\mathrm{n}=17$ ) and those who improved without receiving treatment $(n=41)$. Then, the frequency of other received treatments in these two groups was compared (Table 5) and no significant difference was found between the two groups in this regard except for receiving cell packs. Based on the results (Table 5), 17 (100\%) patients receiving the ophthalmic surgical intervention compared to $31(75.6 \%)$ recovered patients needed pack cell $(P=0.025)$.

Concerning the evaluation of the side effects, only one 26-week-old infant in the intervention group developed bradycardia, thus the administration of propranolol was immediately stopped and the neonate was excluded from the study. The mean duration of hospitalization for intervention and control groups was $43.33 \pm 17.11$ and $45.03 \pm 14.33$ days, respectively, and there was no statistically significant difference between the two groups $(P=0.203)$. Additionally, the mean age of neonates at the time of retinal artery completion in the intervention and control groups was $91.40 \pm 22.16$ and $128.08 \pm 89.79$ days, respectively, indicating no statistically significant difference between the two groups $(P=0.136)$.

Eventually, 28 (96.6\%) patients of the intervention group were discharged, and one (3.4\%) patient was referred to the pediatrics hospital. In the control group, 27 (93.1\%) patients were discharged, one (3.4\%) case died, and one patient $(3.4 \%)$ was referred to the pediatric hospital. Nevertheless, no statistically significant difference was observed between the two groups $(P=0.601)$.

\section{Discussion}

Recent studies have shown that the levels of vascular growth factors, including VEGF, are lower in mice with retinopathy treated with propranolol (8). So far, various studies have focused on the role of beta-blockers in improving retinal neovascularization in animal models $(8,14,15)$. Its proposed mechanism is the blockade of beta-2 adrenoceptors on Müller cells (16). The efficacy of propranolol in preventing angiogenesis is proved in animal models in choroidal neovascularization (14). In recent decades, propranolol administration has been expanded to many neonatal vascular problems (17), and accidentally, it has been observed that it can reduce the incidence and progression of ROP in neonatal hemangiomas (18) and the possible mechanism is through the inhibition of VEGF expression (19). Previous research has also reported a positive association between the 
Table 2. Demographic Findings and Frequency of Other Received Treatments by Patients in the 2 Study Group

\begin{tabular}{|c|c|c|c|c|}
\hline & Intervention Group $(\mathrm{n}=29)$ & Control Group $(n=29)$ & Statistical Test & $P$-value \\
\hline Gestational age (w) & $27.62 \pm 1.54$ & $28.03 \pm 1.65$ & Independent $t$ test & 0.330 \\
\hline Birth weight (g) & $981.03 \pm 222.33$ & $1066.03 \pm 229.36$ & Independent $t$ test & 0.157 \\
\hline APGAR score & $5.51 \pm 2.06$ & $5.00 \pm 2.13$ & Independent $t$ test & 0.353 \\
\hline APGAR score 5 minutes later & $7.41 \pm 1.91$ & $7.13 \pm 1.55$ & Independent $t$ test & 0.550 \\
\hline Neonatal resuscitation at birth & & & Chi-square & \\
\hline Initial steps & $26(89.7)$ & $27(93.1)$ & & 0.640 \\
\hline Positive pressure ventilation & $18(62.1)$ & $21(72.4)$ & & 0.401 \\
\hline Intubation & $5(17.2)$ & $6(20.7)$ & & 0.738 \\
\hline Cardiac massage & 0 & 0 & & - \\
\hline Drugs administration & 0 & 0 & & - \\
\hline Maternal risk factors & & & Chi-square & \\
\hline No risk factor & $16(55.2)$ & $22(75.9)$ & & 0.067 \\
\hline DM & $1(3.4)$ & $1(3.4)$ & & 0.980 \\
\hline PROM & $3(10.3)$ & $1(3.4)$ & & 0.217 \\
\hline Chorioamnionitis & 0 & 0 & & - \\
\hline HTN & $3(10.3)$ & $4(13.8)$ & & 0.109 \\
\hline Hypothyroidism & $5(17.2)$ & $1(3.4)$ & & 0.088 \\
\hline HELLP & $1(3.4)$ & 0 & & 0.780 \\
\hline Steroid administration before birth & $20(68.96)$ & $13(44.8)$ & Chi-square & 0.055 \\
\hline Number of administrations & $1.70 \pm 0.47$ & $1.46 \pm 0.51$ & Independent $t$ test & 0.193 \\
\hline Surfactant administration & $26(89.65)$ & $24(2.75)$ & Chi-square & 0.416 \\
\hline Survanta & $6(20.68)$ & $12(41.37)$ & & 0.231 \\
\hline Curofurf & $11(37.93)$ & $5(17.24)$ & & 0.108 \\
\hline Bles & $9(31.03)$ & $7(24.13)$ & & 0.310 \\
\hline Mechanical ventilation & $6(20.68)$ & $5(17.2)$ & Chi-square & 0.738 \\
\hline Duration (days) & $6.65 \pm 4.49$ & $6.60 \pm 5.45$ & Independent $t$ test & 0.974 \\
\hline Maximum PIP & $19.58 \pm 2.67$ & $20.91 \pm 2.84$ & Independent $t$ test & 0.217 \\
\hline CPAP & $29(100)$ & $29(100)$ & Chi-square & - \\
\hline Duration (days) & $8.34 \pm 4.54$ & $7.06 \pm 5.18$ & Independent $t$ test & 0.323 \\
\hline NIPPV & $6(20.7)$ & $5(17.2)$ & Chi-square & 0.738 \\
\hline Duration (days) & $9.00 \pm 7.18$ & $5.60 \pm 3.20$ & Independent $t$ test & 0.332 \\
\hline HFNC & $29(100)$ & $29(100)$ & Chi-square & - \\
\hline Duration (days) & $13.44 \pm 11.32$ & $12.55 \pm 10.33$ & Independent $t$ test & 0.754 \\
\hline Duration of oxygen therapy (days) & $21.00 \pm 22.00$ & $16.60 \pm 15.64$ & Independent t-test & 0.708 \\
\hline BPD & $27(93.1)$ & $24(82.8)$ & Chi-square & 0.227 \\
\hline Pack cell & $26(89.7)$ & $22(75.9)$ & Chi-square & 0.164 \\
\hline Neonatal age at ROP diagnosis (w) & $47.27 \pm 10.91$ & $50.86 \pm 16.40$ & Independent $t$ test & 0.233 \\
\hline Neonatal weight at ROP diagnosis (gr) & $1529.68 \pm 388.16$ & $1614.09 \pm 180.24$ & Independent t-test & 0.824 \\
\hline
\end{tabular}

Note. DM: Diabetes mellitus; PROM: Prelabor rupture of membrane; HTN: Hypertension; HELLP: High flow nasal cannula; PIP: Peak inspiratory pressure; CPAP: Continuous positive airway pressure; NIPPV: Nasal intermittent positive pressure ventilation; HFNC: High flow nasal cannula; BPD: Bronchopulmonary dysplasia; ROP: Retinopathy of prematurity. 
Table 3. Characteristics of ROP Involvement in Study Patients (Chi-square)

\begin{tabular}{lccc}
\hline & $\begin{array}{c}\text { Intervention } \\
\text { Group }(\mathbf{n = 2 9 )}\end{array}$ & $\begin{array}{c}\text { Control Group } \\
(\mathbf{n = 2 9 )}\end{array}$ & $\boldsymbol{P}$ Value \\
\hline ROP stage & & & \\
1 & $13(44.9)$ & $14(48.2)$ & 0.217 \\
2 & $16(55.1)$ & $15(51.8)$ & \\
ROP zone & & & \\
1 & $2(6.9)$ & 0 & 0.101 \\
2 & $27(93.1)$ & $29(100)$ & \\
3 & 0 & 0 & \\
Involved eye & & & \\
Right & $11(37.9)$ & $12(42.8)$ & 0.183 \\
Left & $14(48.2)$ & $12(42.8)$ & \\
Bilateral & $4(13.9)$ & $5(14.4)$ & \\
\hline
\end{tabular}

Note. ROP: Retinopathy of prematurity.

growth of neonatal hemangiomas and the development of ROP (20), implying the hypothesis that propranolol can play a role in the treatment of ROP by affecting the retinal neovascularization process. On the other hand, neonates and infants well-tolerate the propranolol although, in preterm neonates who are at an increased risk of sepsis, apnea, and bronchopulmonary dysplasia, it can lead to serious complications (21) such as hypotension, bradycardia, bronchial obstruction, and hypoglycemia (22), most of which are dose-dependent (23).
According to our results, the rate of ROP progression in the intervention group was significantly lower than that of the control group. Filippi et al, in a study on the administration of propranolol to treat ROP, reported that the use of oral propranolol can reduce the progression of the disease to stage 3 and stage $3+$ plus, and found that the need for treatment with intravitreal Bevacizumab injection was significantly lower. They further concluded that $19 \%$ of patients developed some side effects including hypotension and bradycardia (22). In another study, they evaluated the efficacy of propranolol in preterm neonates and indicated that neonates in the intervention group had less progress toward stage 3 ROP and needed less laser treatment or intravitreal injection (17). Makhoul et al also showed that the administration of propranolol to treat preterm neonates could reduce the need for invasive treatment in patients with ROP although it was not useful in preventing ROP (24).

Korkmaz et al demonstrated that the administration of propranolol in patients with stage 2 retinopathy significantly reduced the need for laser therapy, but there was no difference in the higher stages of retinopathy (25). Meanwhile, Ozturk and Korkmaz reported that propranolol administration in neonates with stage 2 retinopathy was effective in preventing progression to higher levels although it was not effective in reducing the

Table 4. Rate of ROP Progression in the Two Study Groups

\begin{tabular}{llllll}
\hline & Intervention Group $(\mathbf{n = 2 9 )}$ & Control Group $(\mathbf{n = 2 9 )}$ & P Value & RR $(\mathbf{9 5} \% \mathbf{C l})$ & RRR $(\mathbf{\%})$ \\
\hline Stage $1-2$ ROP progression & $2(15.38)$ & $5(35.7)$ & 0.001 & - \\
Stage 2 - 3 ROP progression & $2(12.5)$ & $9(60.0)$ & 0.009 & $0.416(0.31-0.58)$ & 60 \\
\hline
\end{tabular}

Note. ROP: Retinopathy of prematurity; RR: Risk ratio; RRR: Relative reduction of risk; Cl: Confidence interval.

Table 5. Frequency of Other Treatments in Comparison Between the Group Receiving the Surgical Ophthalmic Intervention (Laser Therapy or Intravitreal Avastin Injection) and Without Ophthalmic Intervention

\begin{tabular}{|c|c|c|c|c|}
\hline & Treated Patients $(n=17)$ & Untreated Patients $(n=41)$ & Statistical Test & $P$ Value \\
\hline Steroid administration before birth & $9(52.9)$ & $24(58.5)$ & Chi-square & 0.695 \\
\hline Number of administration & $1.55 \pm 0.52$ & $1.62 \pm 0.49$ & Independent $t$ test & 0.726 \\
\hline Surfactant administration & $12(70.58)$ & $38(92.68)$ & Chi-square & 0.131 \\
\hline Survanta & $5(41.7)$ & $11(28.9)$ & & 0.130 \\
\hline Curofurf & $6(50.0)$ & $12(31.6)$ & & 0.143 \\
\hline Bles & $1(8.3)$ & $15(39.5)$ & & 0.133 \\
\hline Mechanical ventilation & $4(23.5)$ & $25(61.0)$ & Chi-square & 0.258 \\
\hline Duration (days) & $8.61 \pm 4.92$ & $5.60 \pm 4.46$ & Independent $t$ test & 0.064 \\
\hline Maximum PIP & $20.36 \pm 3.10$ & $20.00 \pm 2.63$ & Independent $t$ test & 0.739 \\
\hline CPAP & $17(100)$ & $41(100)$ & Chi-square & - \\
\hline Duration (days) & $8.82 \pm 5.39$ & $7.24 \pm 4.63$ & Independent $t$ test & 0.265 \\
\hline NIPPV & $2(11.8)$ & $9(22)$ & Chi-square & 0.368 \\
\hline Duration (days) & $7.00 \pm 7.07$ & $7.55 \pm 5.91$ & Independent $t$ test & 0.909 \\
\hline HFNC & $17(100)$ & $41(100)$ & Chi-square & - \\
\hline Duration (days) & $10.41 \pm 6.64$ & $8.71 \pm 4.92$ & Independent $t$ test & 0.075 \\
\hline Duration of oxygen therapy & $24.00 \pm 28.46$ & $16.14 \pm 12.00$ & Independent $t$ test & 0.529 \\
\hline BPD & $16(94.1)$ & $35(85.4)$ & Chi-square & 0.352 \\
\hline Pack cell & $17(100)$ & $31(75.6)$ & Chi-square & 0.025 \\
\hline
\end{tabular}

Note. PIP: Peak inspiratory pressure; CPAP: Continuous positive airway pressure; NIPPV: Nasal intermittent positive pressure ventilation; HFNC: High flow nasal cannula; BPD: Bronchopulmonary dysplasia. 
risk of ROP in stages 0 and 1 (26). Based on the results of Bührer et al, the prophylaxis administration of propranolol did not lead to any difference in the risk of developing ROP in the neonates of the intervention group (27).

It is noteworthy that the probable administration of high oxygen saturation in preterm patients for maintaining sagging in the target range was the reason for increasing the ROP stage in the above-mentioned studies. The findings of another study revealed that reducing the oxygen saturation range in preterm neonates to less than $89 \%$ for decreasing the incidence of ROP can lead to increased hospitalization and neonatal mortality (28). In our study, oxygen saturation was set at $90-95 \%$ in both groups.

Although the results of the current study showed that propranolol administration shortened the completion period of retinal vascularization, there was no statistically significant difference between the two groups, which may be due to the small sample size of the current study, which contradicts the findings of Filippi et al (17).

In the present study, one patient developed adverse events, which led to the discontinuation of propranolol treatment while Makhoul et al and Bancalari et al reported no side effect in separate studies $(24,29)$. On the other hand, in the study by Filippi et al (22), 5 patients (out of 26) presented side effects, which probably is due to the use of a higher dose of propranolol compared to the present study. Therefore, this study introduces $0.5 \mathrm{mg} / \mathrm{kg}$ oral propranolol three times daily as an approximately safe dose for neonates although careful consideration of the possible side effects is strongly recommended as well.

The limited number of patients, due to strict inclusion and exclusion criteria could be a shortcoming of our study. Thus, we recommend more multicenter randomized controlled clinical trials with longer times of follow-up and larger cases to evaluate the efficacy of oral propranolol on the treatment of ROP as opposed to the standard treatment.

Regarding the numerous side effects of the systemic use of beta-blockers, it is recommended that future studies investigate different methods of using beta-blockers (topical and systemic) in patients with ROP in terms of therapeutic efficacy and side effects. It is also suggested that other studies evaluate the serum levels of VEGF, IGF1 , and E-selectin in patients with ROP treated with betablockers and compare them with the control group to investigate possible mechanisms of the drug effects.

\section{Conclusions}

According to the results, propranolol can reduce the rate of ROP progression to higher stages in premature neonates with low BW and thus the need for invasive treatments such as Avastin injection, laser therapy, and vitrectomy. Furthermore, it shortens the completion period of retinal vascularization. Although propranolol can slow down the progression to higher stages, the administration of standard treatments (i.e., laser therapy or intraocular injection of Avastin) should not be delayed because of this finding.

\section{Authors' Contribution}

$\mathrm{MH}$ : writing of proposal, treatment of indicated neonatal by suitable dosage of propranolol, follow up of patients. MMG: management of proposal and paper writing, case selection. MB: writing. SY: Treatment of indicated neonatal with suitable dosage of propranolol, follow up of patients. AEM: Indirect ophthalmoscopy of neonatal, staging of ROP in each patient and follow up of treatment. MRN: Writing.

\section{Conflict of Interests}

Authors have no conflict of interests.

\section{Ethical Issues}

The current study was confirmed by the Ethics Committee of Tabriz University of Medical Sciences (code IR.TBZMED.REC.1397.101) and registered in the Clinical Trial Registration Center of Iran (identifier: IRCT20100512003915N21; https://www.irct.ir/trial/31406). Before recruitment, the study design and its probable safety and efficacy were explained for all parents, and written informed consent was taken accordingly. It was emphasized that participation is voluntary. Data confidentiality was observed during study implementation, no additional cost was imposed on the participants, and the participating neonates were not deprived of standard therapeutic interventions.

\section{Acknowledgments}

Our deepest gratitude goes to all patients in this study and heartfelt thanks go to the health workers of Nikookari and Al-Zahra hospitals.

\section{References}

1. Blencowe $\mathrm{H}$, Cousens $\mathrm{S}$, Chou D, et al. Born too soon: the global epidemiology of 15 million preterm births. Reprod Health. 2013;10(Suppl 1):S2. doi:10.1186/1742-4755-10s1-s2

2. Yau GSK, Lee JWY, Tam VTY, et al. Incidence and risk factors for retinopathy of prematurity in multiple gestations: a Chinese population study. Medicine (Baltimore). 2015;94(18):e867. doi:10.1097/md.0000000000000867

3. Johnson P. Allergic ocular disease: from itchy eyes to visual loss. International Journal of Ophthalmic Practice. 2012;3(1):20-29. doi:10.12968/ijop.2012.3.1.20

4. Patz A. Oxygen administration to the premature infant. A two-edged sword. Am J Ophthalmol. 1967;63(2):351-353. doi:10.1016/0002-9394(67)91567-x

5. Day S, Menke AM, Abbott RL. Retinopathy of prematurity malpractice claims: the Ophthalmic Mutual Insurance Company experience. Arch Ophthalmol. 2009;127(6):794798. doi:10.1001/archophthalmol.2009.97

6. Ferrara N, Davis-Smyth T. The biology of vascular endothelial growth factor. Endocr Rev. 1997;18(1):4-25. doi:10.1210/ edrv.18.1.0287

7. Chen J, Joyal JS, Hatton CJ, et al. Propranolol inhibition of $\beta$-adrenergic receptor does not suppress pathologic neovascularization in oxygen-induced retinopathy. Invest Ophthalmol Vis Sci. 2012;53(6):2968-2977. doi:10.1167/ iovs.12-9691

8. Ristori C, Filippi L, Dal Monte M, et al. Role of the adrenergic system in a mouse model of oxygen-induced retinopathy: antiangiogenic effects of beta-adrenoreceptor blockade. Invest Ophthalmol Vis Sci. 2011;52(1):155-170. doi:10.1167/ iovs.10-5536

9. Afzal A, Shaw LC, Ljubimov AV, Boulton ME, Segal MS, Grant 
MB. Retinal and choroidal microangiopathies: therapeutic opportunities. Microvasc Res. 2007;74(2-3):131-144. doi:10.1016/j.mvr.2007.04.011

10. Schachat AP, Hinton DR, Sadda SR, Wiedemann P. Ryan's Retina. Pediatric Retinal Vascular Diseases. Volume 2. Elsevier; 2013: 1251.

11. Chen J, Stahl A, Hellstrom A, Smith LE. Current update on retinopathy of prematurity: screening and treatment. Curr Opin Pediatr. 2011;23(2):173-178. doi:10.1097/ MOP.0b013e3283423f35

12. Campbell K. Intensive oxygen therapy as a possible cause of retrolental fibroplasia; a clinical approach. Med J Aust. 1951;2(2):48-50. doi:10.5694/j.1326-5377.1951.tb109040.x

13. Martin RJ, Fanaroff AA, Walsh MC. Fanaroff and Martin's Neonatal-perinatal Medicine: Diseases of the Fetus and Infant. Elsevier Health Sciences; 2014.

14. Lavine JA, Sang Y, Wang S, Ip MS, Sheibani N. Attenuation of choroidal neovascularization by $\beta(2)$-adrenoreceptor antagonism. JAMA Ophthalmol. 2013;131(3):376-382. doi:10.1001/jamaophthalmol.2013.1476

15. Ciccarelli M, Sorriento D, Cipolletta $E$, et al. Impaired neoangiogenesis in $\beta_{2}$-adrenoceptor gene-deficient mice: restoration by intravascular human $\beta_{2}$-adrenoceptor gene transfer and role of $\mathrm{NF} \kappa \mathrm{B}$ and $\mathrm{CREB}$ transcription factors. Br J Pharmacol. 2011;162(3):712-721. doi:10.1111/j.14765381.2010.01078.x

16. Martini D, Monte MD, Ristori C, et al. Antiangiogenic effects of $\beta 2$-adrenergic receptor blockade in a mouse model of oxygeninduced retinopathy. J Neurochem. 2011;119(6):1317-1329. doi:10.1111/j.1471-4159.2011.07530.x

17. Filippi L, Cavallaro G, Fiorini $P$, et al. Propranolol concentrations after oral administration in term and preterm neonates. J Matern Fetal Neonatal Med. 2013;26(8):833-840. doi:10.3109/14767058.2012.755169

18. Léauté-Labrèze $C$, Dumas de la Roque $\mathrm{E}$, Hubiche T, Boralevi F, Thambo JB, Taïeb A. Propranolol for severe hemangiomas of infancy. N Engl J Med. 2008;358(24):2649-2651. doi:10.1056/ NEJMc0708819

19. Manunza F, Syed S, Laguda B, et al. Propranolol for complicated infantile haemangiomas: a case series of 30 infants. Br J Dermatol. 2010;162(2):466-468. doi:10.1111/ j.1365-2133.2009.09597.x

20. Praveen V, Vidavalur R, Rosenkrantz TS, Hussain N. Infantile hemangiomas and retinopathy of prematurity: possible association. Pediatrics. 2009;123(3):e484-489. doi:10.1542/ peds.2007-0803

21. Filippi L, Cavallaro G, Fiorini $P$, et al. Study protocol: safety and efficacy of propranolol in newborns with Retinopathy of Prematurity (PROP-ROP): ISRCTN18523491. BMC Pediatr. 2010;10:83. doi:10.1186/1471-2431-10-83

22. Filippi L, Cavallaro G, Bagnoli $P$, et al. Oral propranolol for retinopathy of prematurity: risks, safety concerns, and perspectives. J Pediatr. 2013;163(6):1570-1577.e1576. doi:10.1016/j.jpeds.2013.07.049

23. Lawley LP, Siegfried E, Todd JL. Propranolol treatment for hemangioma of infancy: risks and recommendations. Pediatr Dermatol. 2009;26(5):610-614. doi:10.1111/j.15251470.2009.00975.x

24. Makhoul IR, Peleg O, Miller B, et al. Oral propranolol versus placebo for retinopathy of prematurity: a pilot, randomised, double-blind prospective study. Arch Dis Child. 2013;98(7):565-567. doi:10.1136/archdischild-2013-303951

25. Korkmaz L, Baştuğ O, Ozdemir A, et al. The efficacy of propranolol in retinopathy of prematurity and its correlation with the platelet mass index. Curr Eye Res. 2017;42(1):88-97. doi:10.3109/02713683.2016.1158272

26. Ozturk MA, Korkmaz L. The efficacy of propranolol in very preterm infants at the risk of retinopathy of prematurity: which newborn and when? Int Ophthalmol. 2019;39(9):1921-1930. doi:10.1007/s10792-018-1018-8

27. Bührer C, Erdeve Ö, Bassler D, Bar-Oz B. Oral propranolol for prevention of threshold retinopathy of prematurity (ROPROP): protocol of a randomised controlled trial. BMJ Open. 2018;8(7):e021749. doi:10.1136/bmjopen-2018-021749

28. Stevens TP, Finer NN, Carlo WA, et al. Respiratory outcomes of the surfactant positive pressure and oximetry randomized trial (SUPPORT). J Pediatr. 2014;165(2):240-249.e244. doi:10.1016/j.jpeds.2014.02.054

29. Bancalari A, Schade R, Muñoz T, Lazcano C, Parada R, Peña R. Oral propranolol in early stages of retinopathy of prematurity. J Perinat Med. 2016;44(5):499-503. doi:10.1515/jpm-20150357

Copyright (C) 2022 The Author(s); This is an open-access article distributed under the terms of the Creative Commons Attribution License (http://creativecommons.org/licenses/by/4.0), which permits unrestricted use, distribution, and reproduction in any medium, provided the original work is properly cited. 